\title{
A New Approach for Solving Dual Fuzzy Nonlinear Equations Using Broyden's and Newton's Methods
}

\author{
M. Y. Waziri' ${ }^{1,2}$ and Z. A. Majid'2,3 \\ ${ }^{1}$ Department of Mathematics, Faculty of Science, Bayero University Kano, Kano 3011, Nigeria \\ ${ }^{2}$ Department of Mathematics, Faculty of Science, Universiti Putra Malaysia, 43400 Serdang, Malaysia \\ ${ }^{3}$ Institute for Mathematical Research, Universiti Putra Malaysia, Selangor, 43400 Serdang, Malaysia
}

Correspondence should be addressed to M. Y. Waziri, mywaziri@gmail.com

Received 11 February 2012; Revised 19 July 2012; Accepted 26 July 2012

Academic Editor: Kemal Kilic

Copyright ( $) 2012$ M. Y. Waziri and Z. A. Majid. This is an open access article distributed under the Creative Commons Attribution License, which permits unrestricted use, distribution, and reproduction in any medium, provided the original work is properly cited.

We present a new approach for solving dual fuzzy nonlinear equations. In this approach, we use Newton's method for initial iteration and Broyden's method for the rest of the iterations. The fuzzy coefficients are presented in parametric form. Numerical results on well-known benchmark fuzzy nonlinear equations are reported to authenticate the effectiveness and efficiency of the approach.

\section{Introduction}

Solving systems of nonlinear equations is becoming more essential state in analysis and handling complex problems in many research areas (e.g. robotics, radiative transfer, chemistry, economics, etc.). Consider the nonlinear systems

$$
F(x)=0,
$$

where $F: R^{n} \rightarrow R^{n}$ is a nonlinear mapping. The value of variable $x$ is called a solution or root of the nonlinear equations. The most widest approach to solve such nonlinear systems is Newton's initiative [1], yet it required to compute the Jacobian matrix in every iteration.

However, in some cases, the coefficients of the nonlinear systems are given in fuzzy numbers instead of crisp numbers. Therefore, there is a need to explore some possible numerical methods for solving fuzzy nonlinear equations. It is vital to mention that the basic concept of fuzzy numbers were first presented in [2-4], and the famous application of fuzzy number arithmetic is systems of nonlinear equations in which its coefficients are given as fuzzy numbers $[5,6]$. Moreover, the standard analytical technique presented by
$[7,8]$ cannot be suitable for handing the fuzzy nonlinear equations such as

(i) $a x^{3}+b x^{2}+c x-e=f$,

(ii) $d \sin (x)-g x=h$,

(iii) $i x^{2}+f \cos (x)=a$,

(iv) $x-\cos (x)=d$,

where, $a, b, c, d, e, f, g, h, i$ are fuzzy numbers. In general, we consider these equations as

$$
F(x)=c .
$$

To tackle these situations, some numerical methods have been introduced [8-12]. For example, [11] applied Newton's method while [9] employed Broyden's method and [10] uses steepest descent method to solve fuzzy nonlinear equations, respectively. Nevertheless, the weakness of Newton's method arises from the need to compute and invert the Jacobian Matrix in every iteration.

It worth to mention that [12] has extended the approach of [11] to solve dual fuzzy nonlinear systems. Nevertheless, 
their approach required to compute and store the Jacobian matrix in every iteration. In this paper, a new approach via Newton's and Broyden's methods is proposed to solve dual fuzzy nonlinear equations. The anticipation has been to reduce the computational burden of the Jacobian matrix in every iteration. This paper is arranged as follows: in the next section, we present brief overview and some basic definitions of the fuzzy nonlinear equations, and description of our method is given in Section 3. Section 4 presents the alternative appoach for solving fuzzy nonlinear systems. Numerical results are reported in Section 5, and finally conclusion is given in Section 6 .

\section{Preliminaries}

This section presents some vital definitions of fuzzy numbers.

Definition 1. A fuzzy number is a set like $u: R \rightarrow I=[0,1]$ which satisfies the following conditions [13]:

(1) $u$ is upper semicontinuous,

(2) $u(x)=0$ outside some interval $[c, d]$,

(3) there are real numbers $a, b$ such that $c \leq a \leq b \leq d$ and

(3.1) $u(x)$ is monotonic increasing on $[c, a]$,

(3.2) $u(x)$ is monotonic decreasing on $[b, d]$,

(3.3) $u(x)=1, a \leq x \leq b$.

The set all these fuzzy numbers is denoted by $E$. An equivalent parametric is as also given in [14].

Definition 2 (see [13]). A fuzzy number in parametric form is a pair " $\underline{u}, \bar{u}$ " of functions $\underline{u}(r), \bar{u}(r), 0 \leq r \leq 1$, which satisfies the following:

(1) $\underline{u}(r)$ is a bounded monotonic increasing left continuous function,

(2) $\bar{u}(r)$ is a bounded monotonic decreasing left continuous function,

(3) $\underline{u}(r) \leq \bar{u}(r), 0 \leq x \leq 1$.

See $[11,13,14]$ for more details.

\section{The Classical Broyden's Method}

It is well known that it is not always feasible to compute the full elements of the Jacobian matrix of the given nonlinear function or it may be very expensive, we often have to approximate the Jacobian matrix by some other approaches; the famous method of doing so is quasi-Newton's method [15] and Broyden's method in particular. The basic idea underlining this method has been to reduce the evaluation cost of the Jacobian matrix. Moreover, in some situations analytic derivatives could not be done precisely, or not available to obtain, and the promising method designed to embark upon this situation is Broyden's scheme.
Broyden's method is an iterative procedure that generates a sequence of points $\left\{x_{k}\right\}$ from a given initial guess $x_{0}$ via the following form:

$$
x_{k+1}=x_{k}-B_{k}^{-1} F\left(x_{k}\right) \quad k=0,1,2, \ldots,
$$

where $B_{k}$ is an approximation to the Jacobian which can be updated at each iteration using a rank-one matrix for $k=$ $0,1,2$ and so forth. The appealing feature of this method is that it requires only one function evaluation per iteration. If $F(x)$ is an affine function, and there exist $D \in R^{n \times m}$ and $b \in R^{n \times m}, F(x)=A x+b$; then

$$
F\left(x_{k+1}\right)-F\left(x_{k}\right)=D\left(x_{k+1}-x_{k}\right)
$$

holds. By (4), the updated matrix $B_{k+1}$ is chosen in such a way that it satisfies the secant equation, that is,

$$
B_{k+1} s_{k}=y_{k}
$$

where $s_{k}=x_{k+1}-x_{k}$ and $y_{k}=F\left(x_{k+1}\right)-F\left(x_{k}\right)$.

From (5), the update formulae for the Broyden matrix $B_{k}$ is given as [15]

$$
B_{k+1}=B_{k}+\frac{\left(y_{k}-B_{k} s_{k}\right) s_{k}^{T}}{s_{k}^{T} s_{k}} .
$$

Hence, the number of scalar function evaluations is reduced from $n^{2}+n$ to $n$. In this study, we use classical approach, that is, by using direct inverse $B^{-1}$ instead of approximate $B^{-1}$ used by [9]. In the following, we state the convergence theorems of the Broyden's method, we referred to the proof in [16].

Theorem 3. Let the standard assumptions hold and let $r \in$ $(0,1)$ be given. Then there are $\delta$ and $\delta_{B}$ such that if $x_{0} \in B(\delta)$ and $\left\|E_{0}\right\|_{2}<\delta_{B}$ the Broyden sequence for the data $\left(F, x_{0}, B_{0}\right)$ exists and $x_{k} \rightarrow x^{*}$ q-linearly with $q$-factor at most $r$.

Theorem 4. Let the standard assumptions hold. Then there are $\delta$ and $\delta_{B}$ such that if $x_{0} \in B(\delta)$ and $\left\|E_{0}\right\|_{2}<\delta_{B}$ the Broyden sequence for the data $\left(F, x_{0}, B_{0}\right)$ exists and $x_{k} \rightarrow x^{*}$ q-superlinearly.

\section{Classical Broyden's Method for Solving Dual Fuzzy Nonlinear Equations}

Generally, there exists no inverse of any given fuzzy number, say $x \in E$ such that

$$
x+y=0
$$

where $y \in E$ [17]. In fact, for any nonscrip fuzzy number $x \in E[12]$, it is true that

$$
x+(-x) \neq 0 \text {. }
$$

Here, we consider the dual fuzzy nonlinear system as

$$
Q(x)=R(x)+c,
$$


where all parameters are fuzzy numbers. The basic idea of this section is to obtain a solution for the above dual fuzzy nonlinear equations, whose parametric version is given as

$$
\begin{gathered}
\underline{Q}(\underline{x}, \bar{x}, r)=\underline{R}(\underline{x}, \bar{x}, r)+\underline{c}(r), \\
\bar{Q}(\underline{x}, \bar{x}, r)=\bar{R}(\underline{x}, \bar{x}, r)+\bar{c}(r), \quad \forall r \in[0,1] .
\end{gathered}
$$

Assume that $x=(\underline{\lambda}, \bar{\lambda})$ is the solution to the above fuzzy nonlinear equation, then

$$
\begin{gathered}
\underline{Q}(\underline{\lambda}, \bar{\lambda}, r)=\underline{R}(\underline{\lambda}, \bar{\lambda}, r)+\underline{c}(r), \\
\bar{Q}(\underline{\lambda}, \bar{\lambda}, r)=\bar{R}(\underline{\lambda}, \bar{\lambda}, r)+\bar{c}(r), \quad \forall r \in[0,1] .
\end{gathered}
$$

From (11), we have

$$
\begin{gathered}
\underline{Q}(\underline{\lambda}, \bar{\lambda}, r)-\underline{R}(\underline{\lambda}, \bar{\lambda}, r)=\underline{c}(r), \\
\bar{Q}(\underline{\lambda}, \bar{\lambda}, r)-\bar{R}(\underline{\lambda}, \bar{\lambda}, r)=\bar{c}(r), \quad \forall r \in[0,1] .
\end{gathered}
$$

Letting $\underline{F}(\underline{\lambda}, \bar{\lambda}, r)=\underline{c}(r)$ and $\bar{F}(\underline{\lambda}, \bar{\lambda}, r)=\bar{c}(r)$ in (12), we obtained

$$
\begin{gathered}
\underline{Q}(\underline{\lambda}, \bar{\lambda}, r)-\underline{R}(\underline{\lambda}, \bar{\lambda}, r)=\underline{F}(\underline{\lambda}, \bar{\lambda}, r), \\
\bar{Q}(\underline{\lambda}, \bar{\lambda}, r)-\bar{R}(\underline{\lambda}, \bar{\lambda}, r)=\bar{F}(\underline{\lambda}, \bar{\lambda}, r), \quad \forall r \in[0,1] .
\end{gathered}
$$

To solve (10), an initial guess is required and then generating a sequence of points $\left\{x_{k}\right\}_{k \geq 0}$. Now, we can describe the algorithm for our proposed approach (Broyden's Newton) as follows.

Algorithm 5.

Step 1. Transform the dual fuzzy nonlinear equations into parametric form.

Step 2. Determine the initial guess $x_{0}$ by solving the parametric equations for $r=0$ and $r=1$.

Step 3. Compute the initial Jacobian matrix

$$
J\left(\underline{x}_{0}, \bar{x}_{0}, r\right)=B_{0}(r) .
$$

Using (14), compute $x_{1}$ via Newton's method, hence

$$
x_{1}(r)=x_{0}(r)-B_{0}(r)^{-1} F\left(x_{0}\right)(r),
$$

then compute

$$
F\left(\underline{x}_{1}, \bar{x}_{1}, r\right)=\left[\begin{array}{l}
\underline{F}\left(\underline{x}_{1}, \bar{x}_{1}, r\right) \\
\bar{F}\left(\underline{x}_{1}, \bar{x}_{1}, r\right)
\end{array}\right] .
$$

Step 4. Compute $s_{k}(r)=x_{k}(r)-x_{k-1}(r)$ and $y_{k}(r)=$ $F\left(x_{k}(r)\right)-F\left(x_{k-1}(r)\right)$ to update the current Broyden's matrix for $1 \leq k \leq n$.

Step 5. Compute the next point via Broyden's method.

Step 6. Repeat steps from 3 to 5 and continue with the next $k$ until the stopping criteria $\left(\epsilon \leq 10^{-5}\right)$ are satisfied.

\section{Numerical Results}

In this section, we consider two problems to illustrate the performance of our approach for solving dual fuzzy nonlinear equations. The computations are performed in Matlab 7.0 using double precision computer. The benchmark problems are from [12].

Problem 1 (see [12]). Consider

$$
(2,1,1) x^{3}+(3,1,1) x^{2}+(3+r) x=(4,1,1) x+(4,2,4) .
$$

Without loss of generality, assume $x$ is positive, then we have the parametric equation as [12]

$$
\begin{aligned}
& (1+r) \underline{x}^{3}(r)+(2+r) \underline{x}^{2}(r)+(3+r) \underline{x}(r) \\
& =(3+r) \underline{x}(r)+(2+2 r), \\
& (3-r) \bar{x}^{3}(r)+(4-r) \bar{x}^{2}(r)+(5-r) \bar{x}(r) \\
& =(5-r) \bar{x}(r)+(8-4 r) \text {. }
\end{aligned}
$$

Equivlantly,

$$
\begin{aligned}
& (1+r) \underline{x}^{3}(r)+(2+r) \underline{x}^{2}(r)=(2+2 r), \\
& (3-r) \bar{x}^{3}(r)+(4-r) \bar{x}^{2}(r)=(8-4 r) .
\end{aligned}
$$

We obtained the initial point by letting $r=0$ in (18) as follows:

$$
\begin{aligned}
& \underline{x}^{3}(0)+2 \underline{x}^{2}(0)=2, \\
& 3 \bar{x}^{3}(0)+4 \bar{x}^{2}(0)=8 .
\end{aligned}
$$

For $r=1$, we have

$$
\begin{aligned}
& 2 \underline{x}^{2}(1)+3 \underline{x}(1)=4, \\
& 2 \bar{x}^{2}(1)+3 \bar{x}(1)=4 .
\end{aligned}
$$

We consider the initial guess $x_{0}=(0.8,0.9,0.9)$. By implementing Algorithm 5, the solution was obtained in three iterations with maximum error less than $10^{-5}$. The performance profile of the positive solution for $r \in[0,1]$ is given in Figure 1.

Problem 2 (see [12]). Consider

$$
(6,2,2) x^{2}+(2,1,1) x=(2,1,1) x^{2}+(2,1,1) .
$$

Without loss of generality, let $x$ be positive; hence the parametric form of (22) is given as [12]

$$
\begin{gathered}
(4+2 r) \underline{x}^{2}(r)+(1+r) \underline{x}(r) \\
=(1+r) \underline{x}^{2}(r)+(1+r), \\
(8-2 r) \bar{x}^{2}(r)+(3-r) \bar{x}(r) \\
=(3-r) \underline{x}^{2}(r)+(3-r) .
\end{gathered}
$$




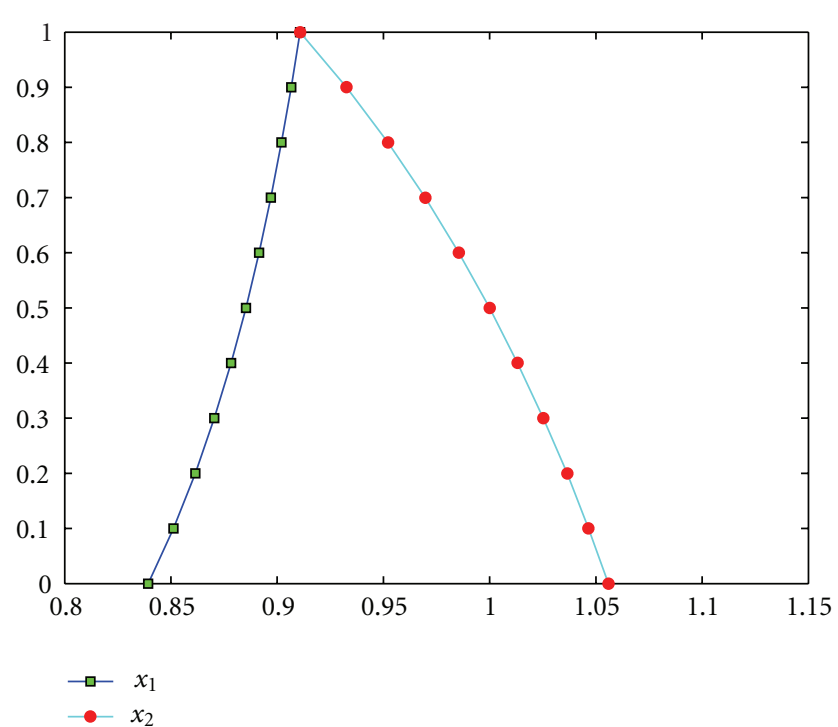

FIgURE 1: Positive solution of the new method for Problem 1.

In other way, (23) can be written as

$$
\begin{aligned}
& (3+r) \underline{x}^{2}(r)+(1+r) \underline{x}(r)=(1+r), \\
& (5-r) \bar{x}^{2}(r)+(3-r) \bar{x}(r)=(3-r)
\end{aligned}
$$

and the Jacobian is

$J(\underline{x}, \bar{x} ; r)$

$$
=\left[\begin{array}{cc}
2(3+r) \underline{x}(r)+(1+r)(r) & 0 \\
0 & 2(5-r) \bar{x}(r)+(3-r)
\end{array}\right] .
$$

Hence, the Jacobian inverse is given as follows:

$$
\begin{aligned}
& J(\underline{x}, \bar{x} ; r)^{-1} \\
& \quad=\left[\begin{array}{cc}
\frac{1}{2(3+r) \underline{x}(r)+(1+r)(r)} & 0 \\
0 & \frac{1}{2(5-r) \bar{x}(r)+(3-r)}
\end{array}\right] .
\end{aligned}
$$

To obtain the initial values, we let $r=0$ and $r=1$ in (23), respectively, therefore

$$
\begin{aligned}
& 3 \underline{x}^{2}(0)+\underline{x}(0)=1, \\
& 5 \bar{x}^{2}(0)+3 \bar{x}(0)=3, \\
& 4 \underline{x}^{2}(1)+2 \underline{x}(1)=2, \\
& 4 \bar{x}^{2}(1)+2 \bar{x}(1)=2 .
\end{aligned}
$$

Thus, from (27), we have $\underline{x}(0)=0.4343, \bar{x}(0)=0.5307$ and $\underline{x}(1)=\bar{x}(1)=0.5000$. Therefore, initial guess $x_{0}=$ $(\underline{x}(0), \underline{x}(1), \bar{x}(0))$. From our own observation, the $x_{0}$ is very close to the solution. Therefore, in order to illustrate the performance of our approach, we consider $x_{0}=(0.4,0.5,0.6)$.

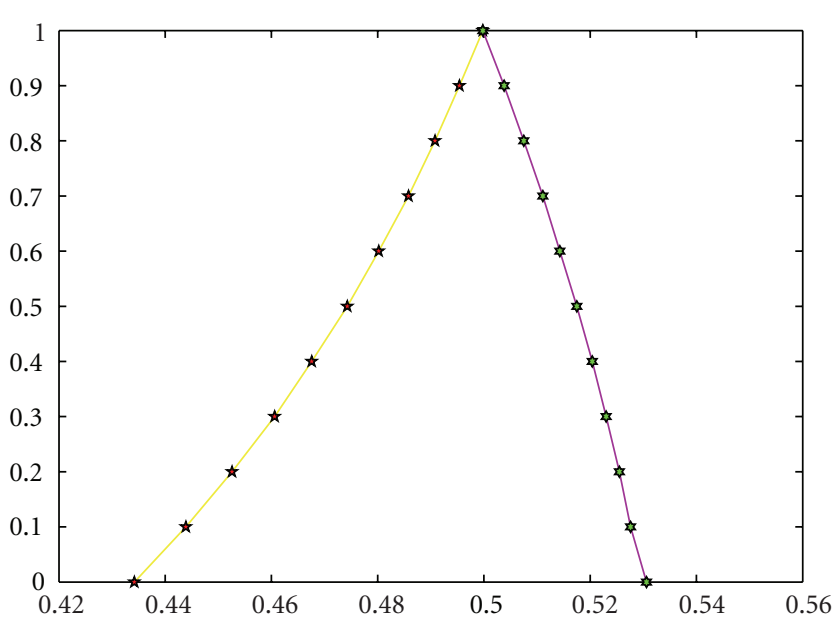

Figure 2: Positive solution of new method for Problem 2.

Via Algorithm 5 with $x_{0}=(0.4,0.5,0.6)$ by repeating 3 to 5 until stopping criterion is satisfied. After three iterations, the solution was obtained with maximum error less than $10^{-5}$. It has been shown in [12] that the negative root of this dual fuzzy nonlinear systems does not exist, that is why we consider only the positive solutions. We present the details of the solution for all $r \in[0,1]$ in Figure 2. Figures 1 and 2 illustrates the efficiency of our approach on solving dual fuzzy nonlinear equations. Our approach converges in three iterations with maximum error less than $10^{-5}$.

\section{Conclusion}

A new approach for solving dual fuzzy nonlinear equations was presented. The approach reduces computational cost of the Jacobian matrix in every iteration. The fuzzy nonlinear equations are transformed into parametric and then solved via Newton's for initial iteration and Broyden's method for rest of the iterations. Numerical experiment shows that in all the benchmark problems, our approach is very promising. Finally, we can claim that our scheme is a good candidate for solving dual fuzzy nonlinear equations.

\section{References}

[1] J. E. Dennis and R. B. Schnabel, Numerical Methods for Unconstrained Optimization and Nonlinear Equations, Prentice Hall, Englewood Cliffs, NJ, USA, 1983.

[2] S. S. L. Chang and L. A. Zadeh, "On fuzzy mapping and control," IEEE Transactions on Systems, Man, and Cybernetics, vol. 2, pp. 30-34, 1972.

[3] D. Dubois and H. Prade, "Operations on fuzzy numbers," International Journal of Systems Science, vol. 9, no. 6, pp. 613626, 1978.

[4] M. Mizumoto and K. Tanaka, "The four operations of arithmetic on fuzzy numbers," Systems Computers and Controls, vol. 7, no. 5, pp. 73-81, 1976.

[5] J. Fang, "On nonlinear equations for fuzzy mappings in probabilistic normed spaces," Fuzzy Sets and Systems, vol. 131, no. 3, pp. 357-364, 2002. 
[6] J. Ma and G. Feng, "An approach to $H_{\infty}$ control off fuzzy dynamic systems," Fuzzy Sets and Systems, vol. 137, no. 3, pp. 367-386, 2003.

[7] J. J. Buckley and Y. X. Qu, "Solving fuzzy equations: a new solution concept," Fuzzy Sets and Systems, vol. 39, no. 3, pp. 291-301, 1991.

[8] J. J. Buckley and Y. Qu, "Solving systems of linear fuzzy equations," Fuzzy Sets and Systems, vol. 43, no. 1, pp. 33-43, 1991.

[9] R. Amira, L. Mohad, and M. Mustafa, "Broyden's method for solving fuzzy nonlinear equations," Advances in Fuzzy Systems, vol. 2010, Article ID 763270, 6 pages, 2010.

[10] S. Abbasbandy and A. Jafarian, "Steepest descent method for solving fuzzy nonlinear equations," Applied Mathematics and Computation, vol. 174, no. 1, pp. 669-675, 2006.

[11] S. Abbasbandy and B. Asady, "Newton's method for solving fuzzy nonlinear equations," Applied Mathematics and Computation, vol. 159, no. 2, pp. 349-356, 2004.

[12] M. T. Kajani, B. Asady, and A. H. Vencheh, "An iterative method for solving dual fuzzy nonlinear equations," Applied Mathematics and Computation, vol. 167, no. 1, pp. 316-323, 2005.

[13] D. Dubois and H. Prade, Fuzzy Sets and Systems: Theory and Application, Academic Press, New York, NY, USA, 1980.

[14] R. Goetschel, Jr. and W. Voxman, "Elementary fuzzy calculus," Fuzzy Sets and Systems, vol. 18, no. 1, pp. 31-43, 1986.

[15] C. G. Broyden, "A class of methods for solving nonlinear simultaneous equations," Mathematics of Computation, vol. 19, pp. 577-593, 1965.

[16] C. T. Kelley, Iterative Methods for Linear and Nonlinear Equations, SIAM, Philadelphia, Pa, USA, 1995.

[17] X. Wang, Z. Zhong, and M. Ha, "Iteration algorithms for solving a system of fuzzy linear equations," Fuzzy Sets and Systems, vol. 119, no. 1, pp. 121-128, 2001. 

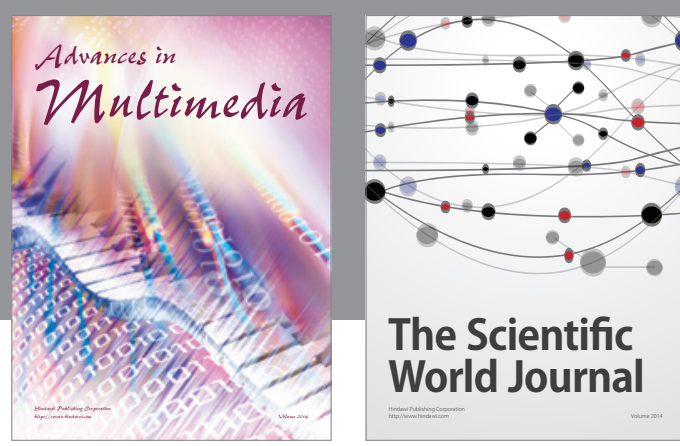

The Scientific World Journal
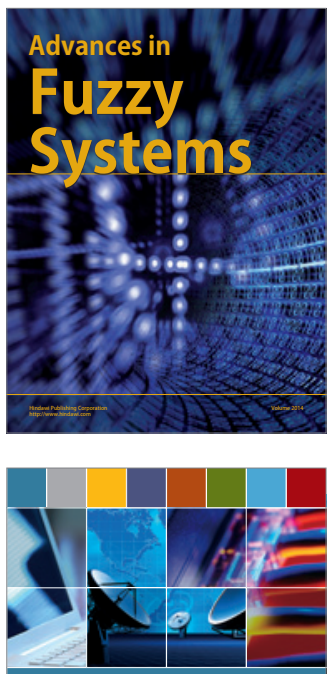

Computer Networks and Communications
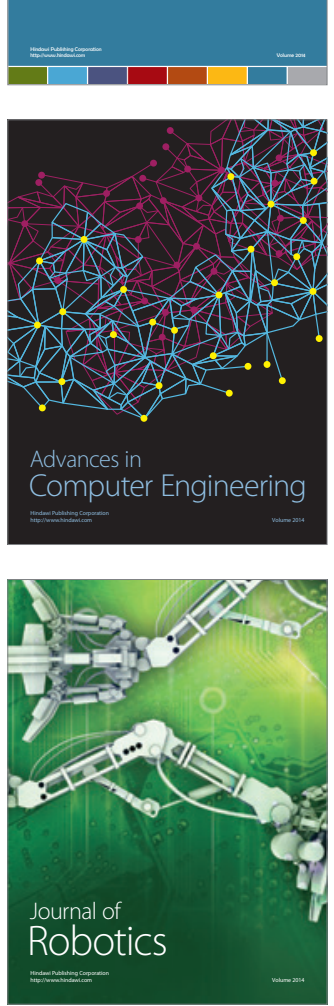
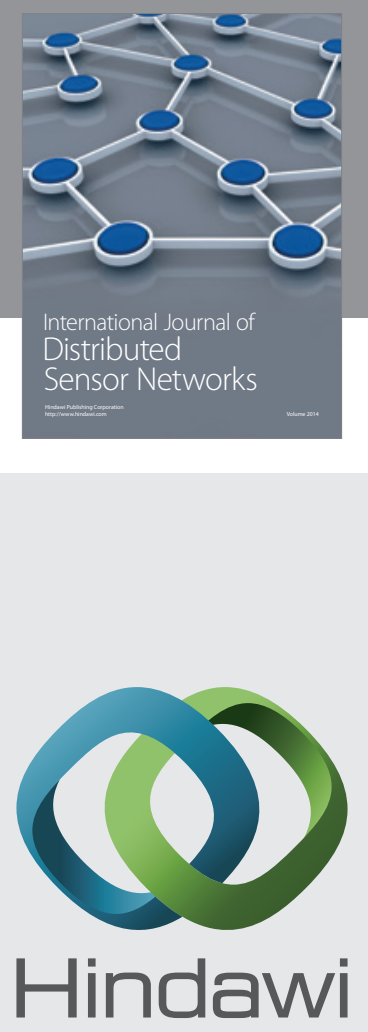

Submit your manuscripts at

http://www.hindawi.com
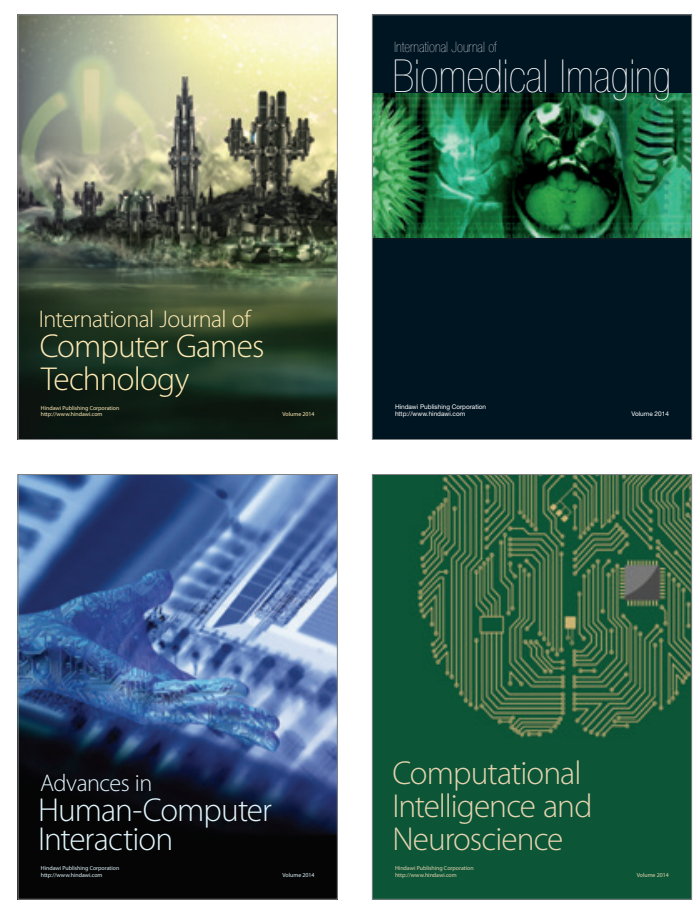
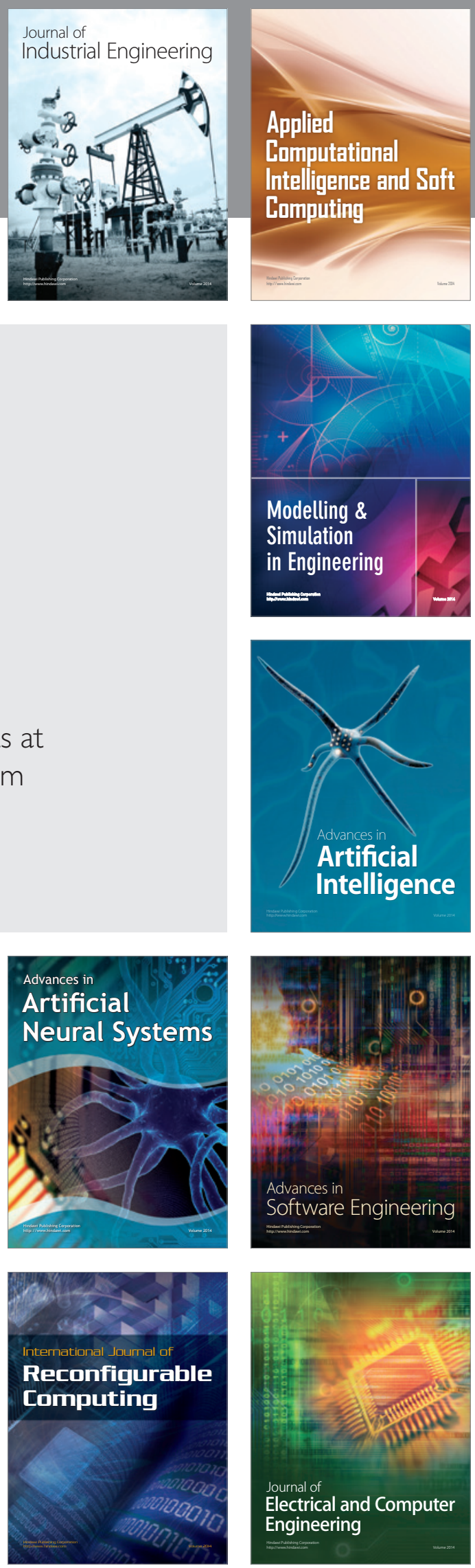